\title{
The clinical value of indocyanine green angiography in the microsurgery of brain cavernomas is very limited
}

\author{
Mika Niemelä • Juri Kivelev • Juha Hernesniemi
}

Received: 5 April 2012 /Accepted: 6 April 2012 /Published online: 3 May 2012

(C) Springer-Verlag 2012

The challenges and potential difficulties in CCM surgery are related mainly to proper patient selection, and thereafter localizing the lesion intraoperatively for safe and effective removal [2]. The removal itself is usually relatively easy due to the known low-flow nature of this vascular malformation shown in previous radiological, surgical, and histopathological studies. Arterial supply presents usually as multiple small-caliber arteries, which are usually non-identifiable in DSA or MRI. Therefore, ICG-VAG is not expected to provide the neurosurgeon with much new useful information to start with.

The often deep location in somewhat eloquent areas restricts the surgical corridor, making the use of ICG-VAG challenging as exposure has to be complete and wide for maximum reliability also in larger multi-lobulated lesions, which may be partly thrombosed. Intraventricular lesions [1] can be visualized well with minimal parenchymal exposure necessary but there were none in the series of Murakami et al. [3]. The authors did not classify their CCMs according to the Zabramski radiological classification based on the stage of intraluminal thrombosis of the lesion [4]. Notably, there are big differences between MR images performed immediately after bleeding and those performed several months later, suggesting somewhat unstable hemodynamics depending on intrinsic and probably extrinsic factors (thrombotic activity of the blood, structural features of the walls of the sinusoids, venous pressure in the region of the CCM, etc.), too. We do not definitely know whether there is always low blood flow within the CCM or whether it exacerbates for some reason, leading to hemorrhage(s). Our understanding

M. Niemelä $(\bowtie) \cdot J$. Kivelev $\cdot$ J. Hernesniemi

Helsinki University Central Hospital,

Topeliuksenkatu, 00260 Helsinki, Finland

e-mail: mika.niemela@hus.fi of hemodynamics in CCM is obviously incomplete, as quantitative data regarding a lesion's inflow and outflow or relationships of the flow between lobules inside the CCM are lacking.

The interpretation of ICG-VAG in CCMs is challenging, and easily with false-negative or false-positive findings, the former being more expected as the neurosurgeon can view only some parts of the surface and some lobules of the CCM. Furthermore, chronic inflammatory processes (typical for CCMs) may stimulate thickening of the wall of the lesion, making interpretation of the ICG unreliable.

During the approach to get enough exposure for ICG, dissection of the lesion from the surrounding parenchyma is necessitated. This often leads to a loss of several feeding arteries, decreasing blood supply to the lesion and consequently affecting intrinsic flow characteristics during the procedure. Additionally, one may misinterpret adjacent small developmental venous malformations as contrast uptake of CCMs during ICG-VAG. Notably, one should not perhaps include CCMs of the orbit in a series of intracerebral lesions since they are a very different entity with different characteristics, natural history, and surgical strategies.

The authors state that "the intraoperative ICG-VAG provides useful information in microsurgical resection of CCMs." The authors have proven with a relatively new method the known fact that CCMs are low-flow vascular malformations, which does have scientific and theoretical value. However, the clinical value is very limited, as ICG-VAG does not help in finding the lesion. Furthermore, CCMs can very well be distinguished intraoperatively from the surrounding parenchyma under the operating microscope by their typical 'mulberry' appearance surrounded typically by yellow hemosiderin. Of importance, the extent of removal of a CCM cannot by reliably estimated by using ICG-VAG. 


\section{Conflicts of interest None.}

\section{References}

1. Kivelev J, Niemelä M, Kivisaari R, Hernesniemi J (2010) Intraventricular cerebral cavernomas: a series of 12 patients and review of the literature. J Neurosurg 112(1):140-149
2. Kivelev J, Niemelä M, Hernesniemi J (2012) Treatment strategies in cavernomas of the brain and spine. J Clin Neurosci 19(4):491-497

3. Murakami K, Endo T, Tominaga T (2012) An analysis of flow dynamics in cerebral cavernous and orbital cavernous angioma using indocyanine green videoangiography. Acta Neurochir (in press)

4. Zabramski JM, Wascher TM, Spetzler RF, Johnson B, Golfinos J, Drayer BP, Brown B, Rigamonti D, Brown G (1994) The natural history of familiar cavernous malformations: results of an ongoing study. J Neurosurg 80(3):422-432 